\title{
La Adquisición del Léxico del Inglés Comercial Mediante un Modelo Computacional y la Efectividad del Uso de Asociaciones Sintagmáticas
}

\author{
Ángela Ma Larrea EsPinar \\ Facultad de Derecho y Ciencias Económicas y Empresariales \\ Universidad de Córdoba
}

Recibido: 3 junio 2009 / aceptado 11 enero 2010

ISSN: $1697-7467$

\begin{abstract}
RESUMEN: Este artículo resume una intervención experimental llevada a cabo con la finalidad didáctica de impulsar el aprendizaje léxico del inglés comercial. Una vez señalada la primacía del componente léxico en este campo, nuestro estudio pretende determinar si el uso de las nuevas tecnologías (TICs en adelante) a través del diseño de materiales propios secuenciados cognitivamente incide positivamente en el aprendizaje léxico. Asimismo, hemos destacado la relevancia de las conexiones sintagmáticas planteando la posibilidad de que la adquisición de vocabulario mediante colocaciones sea más sólido y funcional que el aprendizaje de elementos de manera aislada. Los resultados obtenidos confirman nuestra primera predicción, mostrando una ostensible mejora en la adquisición léxica y apuntan a la validez de la segunda hipótesis.

Palabras clave: Inglés comercial, colocaciones, asociaciones sintagmáticas, adquisición léxica, TICs.
\end{abstract}

The acquisition of lexis of commercial English through a computational model and the effectiveness of use of sitagmatic associations

\begin{abstract}
This article summarizes an experimental study carried out to encourage the learning of Business English lexis, as the key element of this professional area. Firstly, we investigated the hypothesized advantage of designing specific materials following a cognitive-based structure. Secondly, we pointed at the relevance of syntagmatic relations in the structure of the L2 mental lexicon. If this is the case, learning lexical collocations could be proved more useful and lasting than learning isolated elements. The results support the first hypothesis, showing that the students improved extensively their lexical knowledge after the experiment. In relation to the second question, it was found out that not all the students learnt more lexical collocations although the number was low enough not to rule out our assumption and encourage further experiments.

Keywords: Business English, collocations, syntagmatic relations, lexical acquisition, ICTs.
\end{abstract}




\section{InTRODUCCIÓN}

La idea que configura este artículo surge de la praxis docente como profesora de la materia de inglés comercial ante la manifiesta dificultad del alumnado para adquirir el vocabulario específico del campo comercial en inglés ${ }^{1}$.

Así, observamos que la mayoría de los estudiantes de inglés de negocios son neófitos en esta disciplina. Siendo éste el caso, el grado de complejidad de la materia se eleva, ya que a la dificultad de la disciplina propia (llamémosla comercial), se añade el escollo lingüístico. Es decir, al tratarse de una asignatura académica, lo más usual es que el alumnado no tenga experiencia laboral alguna, por lo que no sólo debe adquirir el lenguaje específico, sino también los conceptos de la disciplina ${ }^{2}$.

Aunque el lenguaje especializado se erige sobre el inglés general y comparte destrezas y estrategias de aprendizaje (Hutchinson y Water, 1980; Kitkauskiené 2006), nuestros estudiantes se ven desbordados más que por la terminología técnica del sublenguaje comercial, por la variabilidad de significado que presentan los términos con respecto al inglés general.

Éste es el motivo por el que decidimos diseñar una intervención experimental a través de un programa virtual creado específicamente para fomentar el aprendizaje funcional de vocabulario comercial. Nuestro estudio plantea dos interrogantes que fueron posteriormente analizados en virtud de los resultados obtenidos. Este artículo sintetiza el experimento llevado a cabo y las conclusiones extraídas.

\section{Marco teÓRICO}

\subsection{Las TICs como elemento facilitador de la enseñanza}

Las nuevas tecnologías han adquirido una importancia decisiva en el marco teórico y práctico de la enseñanza de idiomas. Este hecho no tiene un cariz novedoso ya que antes del cambio de siglo eran numerosos los estudios que predecían o confirmaban la jerarquía de la enseñanza virtual computerizada.

Como era de esperar, todas estas predicciones se han cumplido y, de la informática como asignatura que apuntaba a la alfabetización del alumno para manejar el lenguaje de los ordenadores, pasamos a la formación a nivel de usuario, esto es, a aprender a manejar las herramientas computacionales (procesadores de textos, hojas de cálculo, etc.)

No obstante, el proceso de evolución no se detuvo aquí y, en unos años, el ordenador pasó de objeto de estudio a instrumento mediador o facilitador de la enseñanza, ampliando sus utilidades.

Hoy en día, es este aspecto (el de herramienta de apoyo al proceso de enseñanza y aprendizaje) el más relevante por ser extremadamente útil y práctico.

\footnotetext{
${ }^{1}$ La autora de este artículo imparte docencia en la Facultad de Derecho y Ciencias Económicas y Empresariales de la Universidad de Córdoba.

${ }^{2}$ Pickett (1988:90) se refirió a esto en su día como la diferencia entre «knowing about something« y «acting«. Otros términos con el mismo sentido son pre-service y in-service learners (Brieger 1997:12).
} 
Es, pues, un hecho obvio que las crecientes posibilidades de las aplicaciones informáticas han modificado espectacularmente las formas de enseñar y aprender. Atrás quedaron los años de las transparencias, el papel de calco y el radio-casete.

En la actualidad, las TICs ofrecen una gama de recursos hasta hace poco insospechada, y el ordenador se erige en el centro de esta multiplicidad de opciones: DVD, CD-ROM, Internet, vídeo conferencia, teleformación ... sin olvidar el diseño de material interactivo específico.

Para el alumno que aprende un idioma, las aplicaciones informáticas constituyen un manantial de opciones teóricas y prácticas, lingüísticas y culturales. De aquí el valor incuestionable de las TICs en inglés, especialmente dentro de un campo específico.

Creemos que las TICs pueden facilitar extraordinariamente el arduo aprendizaje de un área específica como el inglés comercial y, en particular, impulsar el aprendizaje funcional de la terminología propia del área, que es uno de los elementos más costosos de enseñar como señalábamos anteriormente.

En esta línea, empleamos un soporte informático para diseñar una serie de actividades interactivas cognitivamente estructuradas. Es decir, los ejercicios están secuenciados en función de las distintas etapas del proceso de adquisición léxica (deducción, consolidación, refuerzo y producción):

- Aprehensión de las características del término

- Almacenaje y configuración en la red del léxico mental

- Consolidación y reforzamiento del término mediante repetidas exposiciones para aprehender la totalidad de su alcance cognitivo

- Uso funcional del vocabulario adquirido mediante un proceso interactivo

Además, al diseñar nuestros propios materiales recreando las operaciones mentales del proceso de adquisición de vocabulario intentamos lograr un doble objetivo:

- incrementar la exposición a los términos, dinamizando la incidentalidad.

- afianzar la adquisición funcional y la retención a largo plazo.

Por consiguiente, en lo que respecta a esta primera cuestión, nuestro experimento intentará demostrar que un proceso de aprendizaje de vocabulario formal y teóricamente fundamentado debe ser suficiente para mejorar la adquisición léxica.

\subsection{La trascendencia de las conexiones sintagmáticas en el aprendizaje de vocabulario}

Nuestro segundo interrogante investiga la relevancia del aprendizaje de vocabulario mediante colocaciones léxicas.

El valor conferido al léxico en este artículo no emana sólo de la práctica educativa, sino que se ampara en el redescubierto enfoque léxico en la enseñanza de idiomas y, principalmente, en la centralidad del componente léxico en el inglés comercial.

Siendo el inglés comercial una parcela muy amplia del ESP (Nelson: 2000:3.3), es precisamente la complejidad de esta disciplina la que determina la primacía del léxico. Así, para Pickett el inglés comercial es un ergolecto (lenguaje de trabajo) y esto implica la preponde- 
rancia del componente léxico sobre el gramatical: «An ergolect operates at the level of lexis and at the level of transaction, hardly at all at the level of grammar». (Pickett, 1989:11)

En otras palabras, el léxico es la clave del inglés comercial, lo que lo diferencia de otras disciplinas. Si nuestros alumnos no dominan el léxico comercial, difícilmente podrán desenvolverse adecuadamente en el mundo de los negocios.

En el panorama léxico, las últimas investigaciones se centran en el estudio de vocabulario más allá del término individual, de la palabra en sí: colocaciones, agrupaciones de palabras y frases hechas ${ }^{3}$.

En su acepción más básica: «the collocations of a word are the company that it keeps» (Nation, 1990:32). Así, el término colocación hace referencia a las palabras que suelen acompañar a un término. Carter ofrece una explicación más concreta:

Collocation is a term used to describe a group of words which occur repeatedly in a language. These patterns of co-occurrence can be grammatical in that they result primarily from syntactic dependencies or they can be lexical in that, although syntactic relationships are involved, the patterns result from the fact that in a given linguistic environment certain lexical items will co-occur. (2000:51)

La trascendencia del concepto de colocación en lo referente al aprendizaje de vocabulario se pone de manifiesto en los siguientes puntos (Nation, 2001:318):

1. Language knowledge is collocational knowledge.

2. All fluent and appropriate language use requires collocational knowledge.

3. Many words are used in a limited set of collocations and knowing these is part of what is involved in knowing the words.

Por tanto, la clave para hablar un idioma fluidamente se encuentra en las secuencias de palabras almacenadas en nuestra memoria; sólo a través de la fluidez (sin necesidad de elaboración previa) se alcanza la competencia lingüística: In effect, the mind uses an abundant resource (long-term memory) to store a number of prefabricated chunks of language that can be used 'ready-made' in language production. (Conklin and Schmitt, 2008: 75)

Con respecto a las colocaciones, Miller (1999) manifiesta que un aspecto esencial del conocimiento de una palabra es la capacidad de aludir a una representación cognitiva del tipo de contextos en que ese término adquiere un significado específico. Este conocimiento contextual se compone de: local context, topical context y situational context (Nation, 2001:57). El contexto local no es sino la información proporcionada por las palabras con las que el término se relaciona a nivel sintagmático, es decir, las colocaciones. En referencia al contexto temático y situacional, la centralidad del ámbito, en nuestro caso el área comercial, es indiscutible. En esta línea, el concepto de colocación está íntimamente vinculado a una correcta interpretación y al uso productivo del vocabulario.

La importancia de las conexiones sintagmáticas encuentra también su explicación en las investigaciones llevadas a cabo en el área de la configuración del léxico mental. Así, los resultados obtenidos a través de experimentos asociativos apuntan a que los términos se

${ }^{3}$ Proponemos las siguientes equivalencias en inglés aunque advertimos que la terminología varía según los autores: collocations, multi-word items y prefabricated phrases. 
organizan en campos semánticos en nuestra mente. En relación a la L1, los dos tipos de vínculos asociativos más sólidos son las conexiones entre colocaciones y entre términos coordinados. Igualmente, hay indicios que apuntan a una estructuración léxica diferente en la L1 y la L2. Las observaciones de Meara (1996) y Wolter (2001) sugieren el predominio de asociaciones sintagmáticas en la L2. Si estos hallazgos son correctos y los vínculos sintagmáticos soportan el peso organizativo del lexicón de la L2, la adquisición léxica mediante colocaciones debería ser más efectiva.

Siguiendo este planteamiento, nuestro experimento pretende analizar si el aprendizaje mediante asociaciones sintagmáticas es más duradero y funcional.

\section{Metodología}

Si los estudiantes realizan un curso virtual con ejercicios diseñados por nosotros y secuenciados metodológicamente según un proceso de aprendizaje teóricamente fundamentado, la adquisición léxica debe mejorar ostensiblemente.

Esta hipótesis fue testada comparando los resultados de los pre-tests y de los post-tests realizados por los alumnos.

Por otra parte, si el lexicón de la L2 se estructura principalmente mediante conexiones sintagmáticas, el aprendizaje léxico mediante colocaciones debe conducir a una adquisición más sólida y funcional.

Esta hipótesis fue comprobada analizando las respuestas correctas y verificando si los elementos léxicos habían sido aprendidos mediante colocaciones o de manera aislada (definición o sinónimo).

La existencia de 4 grupos experimentales con distintas unidades léxicas refuerza las conclusiones extraídas y permite estudiar los resultados desde múltiples perspectivas, como por ejemplo la incidencia del tipo de aprendizaje en la adquisición.

\subsection{La selección del corpus}

Para obtener el vocabulario adecuado que nos permitiera desarrollar los ejercicios, nos hacía falta el respaldo de un corpus. Éste era el punto de partida para, a partir de ahí, elaborar una lista de palabras representativas del inglés comercial. No obstante, la estructuración del corpus no era un fin en sí mismo para nuestro proyecto, sino un medio ya que nuestra intención es mejorar la adquisición de vocabulario comercial, no determinar este léxico. Por esta razón, tomamos la decisión de utilizar un corpus ya creado, para poder dedicar así el grueso de nuestro esfuerzo al diseño de actividades y análisis de los resultados de los grupos experimentales.

Así, hemos usado como punto de partida en nuestra tesis, el trabajo realizado por Mike Nelson, profesor de la Universidad de Turku, Finlandia ${ }^{4}$. Nelson creó dos corpus para su estudio: uno de materiales publicados y otro de inglés comercial ${ }^{5}$ y empleó como referencia

\footnotetext{
${ }^{4}$ Para más información, véase: http://users.utu.fi /micnel/ business _ english_lexis_site.htm. (Última consulta: mayo 2008).

${ }^{5}$ Se pueden consultar en la dirección anterior.
} 
el corpus de muestra del BNC (British National Corpus). A continuación, llevó a cabo la comparación léxica entre los tres corpus y, con la ayuda de la herramienta Wordsmith Tools 3 (Scott, 1999), procedió a determinar el léxico característico del lenguaje comercial entre otra serie de aplicaciones.

Las listas de palabras confeccionadas por este autor constituyen un punto de referencia fundamental al combinar la noción de frecuencia con la de término clave ${ }^{6}$. Decidimos, pues, emplear su lista de palabras clave positivas del corpus de inglés comercial, más concretamente la lista de 50 palabras clave?

Las razones que nos llevaron a seleccionar esta lista de palabras han sido múltiples:

- las palabras de esta serie pertenecen a varias categorías gramaticales, no a una en concreto.

- los vocablos están organizados en grupos semánticos.

- las unidades léxicas de cada grupo representan distintos niveles de frecuencia, es decir, no son por ejemplo los 10 primeros verbos más frecuentes ${ }^{8}$.

- se adjunta un estudio individual de cada palabra. Nelson analiza cada término exhaustivamente de acuerdo con varias nociones. Aquí presentamos sólo las que nos han servido de ayuda para la preparación de actividades:

- prosodia semántica (los grupos léxico-semánticos con los que ese término suele combinarse)

- 3-word clusters (grupos de tres palabras de los que forma parte el vocablo)

- colligation (patrones gramaticales de esa palabra, distintos sentidos y relación de la estructura gramatical con el significado si la hay)

\subsection{Diseño y tratamiento de los materiales}

Tal y como hemos avanzado, manejamos la lista de 50 palabras clave confeccionada por Nelson como punto de partida para la selección de vocabulario que se introduciría en los ejercicios. A nuestro parecer, 50 palabras era un número demasiado amplio de vocablos, por lo que escogimos 24 términos al azar y con distinto nivel de frecuencia para obtener una muestra variada y aleatoria que supusiese un grupo léxico representativo para el aprendizaje del alumnado.

El siguiente cuadro representa la categorización semántica de los términos que empleamos en nuestro estudio:

${ }^{6}$ Los términos que se usan con una frecuencia extraordinaria en inglés comercial (siempre en comparación con el inglés general) se denominan palabras clave positivas (positive key words); mientras que aquellos términos que se presentan en inglés comercial con menor frecuencia, y de manera menos significativa que en inglés general, se convierten en palabras clave negativas (negative key words).

7 Nelson, 2000: http://users.utu.fi/micnel/BEC/keywordsindex.htm. (Última consulta: mayo 2008). Esta lista aparece recogida en el anexo 3.

${ }^{8}$ No necesitamos que todas las palabras que vamos a proponer para nuestro estudio sean las primeras en la lista de frecuencia, ya que nuestro propósito es intentar mejorar el aprendizaje de vocabulario. Así, si las palabras son las más frecuentes, siempre se podría aducir que los alumnos las han aprendido mejor porque son las que ven más frecuentemente y son las más conocidas. 
Tabla 1: Categorización semántica de los términos de nuestro estudio.

\begin{tabular}{|l|l|l|l|l|}
\hline $\begin{array}{l}\text { People in } \\
\text { Business }\end{array}$ & $\begin{array}{l}\text { Business } \\
\text { Activities }\end{array}$ & $\begin{array}{l}\text { Business } \\
\text { Actions }\end{array}$ & $\begin{array}{l}\text { Business } \\
\text { Descriptions }\end{array}$ & $\begin{array}{l}\text { Business Events } \\
\text { and Entities }\end{array}$ \\
\hline customer & business & manage & low & sale \\
\hline manager & competition & provide & global & trade \\
\hline supplier & takeover & send & competitive & package \\
\hline distributor & distribution & achieve & strategic & service \\
\hline staff & & & & earnings \\
\hline boss & & & & product \\
\hline
\end{tabular}

A continuación, distribuimos los términos en tres grupos, cada uno de ellos compuesto de 8 palabras con representación de los cinco grupos semánticos:

Tabla 2: Grupos de palabras.

\begin{tabular}{||l|l|l||}
\hline \hline GRUPO LEXXICO 1 & GRUPO LEXXICO 2 & GRUPO LEXICO 3 \\
\hline distributor & supplier & customer \\
\hline boss & staff & manager \\
\hline competition & business & takeover \\
\hline distribution & provide & send \\
\hline manage & achieve & competitive \\
\hline low & global & strategic \\
\hline sale & trade & package \\
\hline earnings & service & product \\
\hline \hline
\end{tabular}

Estos conjuntos de palabras, nos sirvieron para proponer 4 secuencias de estudio, cada una de las cuales se asoció posteriormente a un grupo experimental de estudiantes.

\subsubsection{Secuencia 1}

Esta secuencia es diferente a las demás y es la más compleja, ya que hemos empleado los tres grupos de términos para el diseño de las actividades. El objetivo es que el alumno estudie y practique todas las palabras introducidas en el estudio general.

No obstante, cada grupo de términos se presenta de una manera concreta, por lo que el aprendizaje de vocabulario se considera desde tres perspectivas diferentes: definiciones, sinónimos y colocaciones. Así, las palabras del primer grupo léxico se introducen mediante la descripción de ese término en inglés por lo que, tras la realización de cinco ejercicios secuenciados metodológicamente, el estudiante debe a aprender el significado de los términos a través de su definición.

Seguidamente, el alumnado procederá al aprendizaje de los términos del grupo léxico 2 mediante sinónimos. Otras cinco actividades estructuran la adquisición de estos vocablos.

Finalmente, el estudiante aprenderá dos colocaciones vinculadas a cada uno de los términos del grupo 3 (por ejemplo: customer-driven y customer service) ${ }^{9}$. Queremos destacar

${ }^{9}$ La determinación de las dos colocaciones llevadas a estudio se ha basado en el análisis individual de las palabras realizado por el profesor Nelson (la prosodia semántica, los grupos de palabras frecuentes 
que el alumno no llega a aprender el significado de los vocablos de este grupo léxico de manera individual, ya que únicamente se le presentan dos colocaciones que contienen el término en sí. En esta ocasión, son seis los ejercicios propuestos para el aprendizaje de las colocaciones $^{10}$.

Los estudiantes trabajan con un total de 32 términos (el último grupo cuenta doble al presentarse dos colocaciones por unidad léxica) ${ }^{11}$.

Figura 1: Ejemplo de ejercicio de Matching

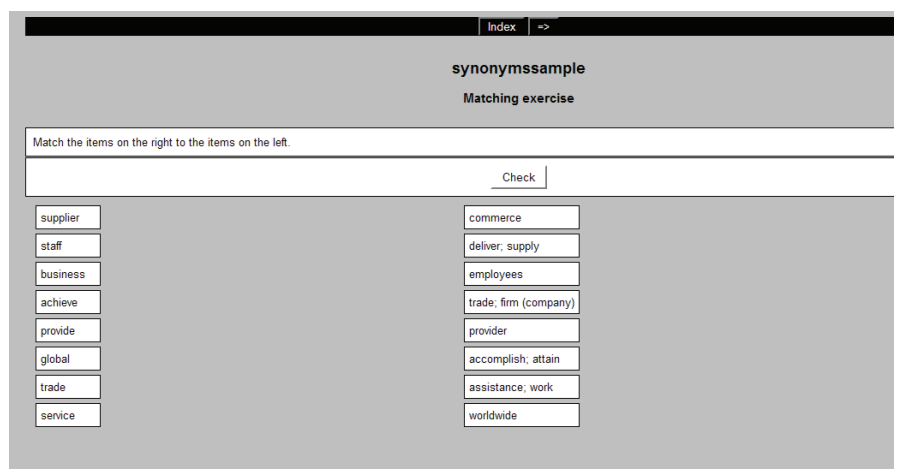

\subsubsection{Secuencias 2, 3 y 4}

El planteamiento de las actividades de estas secuencias va encaminado a la enseñanza de un único grupo de palabras haciendo uso de las tres perspectivas de aprendizaje que hemos planteado anteriormente: definiciones, sinónimos y colocaciones.

De esta manera, en la secuencia 2 los alumnos trabajarán el primer grupo léxico. Vamos a introducir diferentes maneras de asimilar el significado. Por esta razón, elaboramos tres grupos de actividades: el primero trabaja con la definición del término en inglés (4 ejercicios), el segundo con un sinónimo (4 actividades) y el tercero ofrece dos colocaciones (5 actividades) $)^{12}$.

La secuencia 3 se ocupa de los términos del grupo léxico 2 y es similar en planteamiento y estructuración a la secuencia anterior.

(clusters) y la compañía sintáctica (colligation), aunque también hemos tratado de introducir colocaciones fijas o recurrentes que están lexicalizadas y vienen recogidas en el diccionario. De este modo, hemos intentado atender a tanto a criterios de frecuencia como de representatividad.

${ }^{10}$ Las actividades diseñadas con el programa Hot Potatoes están conformadas por: Matching (para el aprendizaje de colocaciones emplearemos 2 ejercicios), Crossword, Short Answer o Quiz (respuesta corta u opción múltiple), Cloze (se proponen 2 ejercicios de rellenar huecos).

${ }^{11}$ Nos gustaría puntualizar que los estudiantes no llegan a aprender el significado de los vocablos del grupo léxico 3 de manera individual, ya que únicamente se le presentan dos colocaciones que contienen el término en sí.

${ }^{12}$ En este caso, las actividades a las que nos referimos son: Matching, Crossword, Quiz y Cloze (con un doble ejercicio de Matching para las colocaciones). 
La secuencia número 4, repite la configuración de las anteriores secuencias con diferentes elementos léxicos, los correspondientes al grupo 3 .

Cada una de estas secuencias se compone de 24 elementos léxicos: 8 términos y 2 colocaciones asociadas a ellos.

Figura 2: Planteamiento de actividades para las secuencias 2, 3 y 4 .

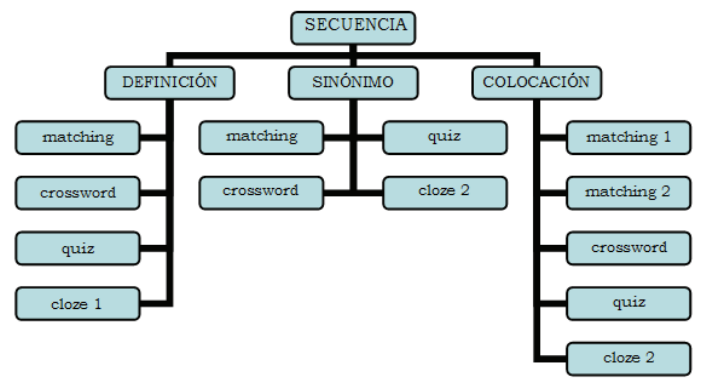

En resumen, las pruebas diseñadas para las secuencias 2, 3 y 4 persiguen que el alumnado aprenda desde varias perspectivas y de manera funcional el vocabulario asignado.

\subsection{Instrumentos}

En este apartado procederemos a revisar y a describir las herramientas que hemos empleado para nuestro estudio experimental y para analizar los datos obtenidos: pre-tests, CDs, posttests, cuestionario personal y autoevaluación.

\subsubsection{Pre-tests}

Con la ayuda de esta herramienta determinaremos el grado de conocimiento previo del alumnado. Hemos diseñado 4 pre-tests que corresponden a las 4 secuencias de estudio descritas previamente. No obstante, el formato es similar:

- encabezamiento información personal ${ }^{13}$

- léxico objeto del estudio

- frases con huecos para completar con los elementos léxicos indicados (similar al Cloze $)^{14}$

${ }^{13}$ Esta información (curso, licenciatura y calificación si ha cursado otra asignatura de inglés comercial anteriormente) ha sido requerida a todos los participantes para constatar la diversidad académica y personal de los integrantes de cada grupo.

${ }^{14}$ Las oraciones que constituyen el pre-test son frases reales, recopiladas de textos económicos y comerciales que se recogen en la página web del periódico Financial Times. También son únicas, en el sentido en que no se han reutilizado para las actividades deCloze propuestas en las distintas secuencias programadas para el estudio del alumno. 
Figura 3: Pre-test 2, correspondiente a la Secuencia 2

Name:

Current course:

Previous course(s)/ year/ grade:

Expected degree:

PRE-TEST CLOZE 2

Fill in all the gaps with a suitable word from the box:

\begin{tabular}{|l|}
\hline competition competition competition sale sale sale \\
distributors distributor distributor manage manage manage \\
earnings earnings earnings boss boss boss low low low \\
distribution distribution distribution
\end{tabular}

1. Greg Gailey, chief executive of Australia's Zinifex, said prices were expected to remain well above long-term averages given hefty global demand and ................... inventory levels.

2. Analysts believe that the latest round of price rises could signal the end of a period of aggressive ................. among supermarkets a retailers act to restore profit margins and respond to rising costs.

3. It occupies about one-eighth of the space of a regular desktop PC, has a ...............end Intel processor and comes with Microsof

4. The CBI said the companies that saw the biggest improvement were those making goods for direct ...................... to consumers, such as those making goods for direct .....................

5. Some industry experts supported the government's decision, arguing that its plan for competitive electricity ..................... was misconceived.

\subsubsection{Post-tests}

Serán esenciales para determinar el grado de aprendizaje del alumno, por lo que funciona como actividad experimental. El post-test no es otro que el mismo ejercicio que hemos elaborado bajo el nombre de pre-test, sólo que, en este caso, el estudiante lo realiza tras haber completado las actividades propuestas para su grupo. Teniendo en mente nuestros fines didácticos, no hemos estimado oportuno elaborar un ejercicio distinto al pre-test para evitar desvirtuar los resultados. Es decir, utilizando las mismas frases, el grado de dificultad de las mismas no se acrecienta ni disminuye. Cada alumno volverá a completar el test tras haber realizado los ejercicios correspondientes a su grupo.

\subsection{3. $C D$}

Tras haber elaborado las cuatro secuencias de actividades, hemos procedido a la creación de los respectivos CDs interactivos para el aprendizaje personal, uno por cada secuencia de ejercicios.

Hot potatoes $^{t m}$, programa de enseñanza interactiva, nos permite confeccionar una página web para realizar las actividades. De esta manera, hemos diseñado una página web a modo de índice para cada una de las series creadas. Aquí aparecen desglosados los ejercicios que 
conforman cada secuencia de estudio y que el alumno deberá llevar a cabo en el mismo orden propuesto.

En último lugar, hemos vinculado cada uno de los ejercicios para que el estudiante, con un doble clic, pueda acceder a ellos rápidamente desde cualquier ordenador, sin necesidad de conexión a Internet.

Figura 4: Índice e instrucciones del $C D$.

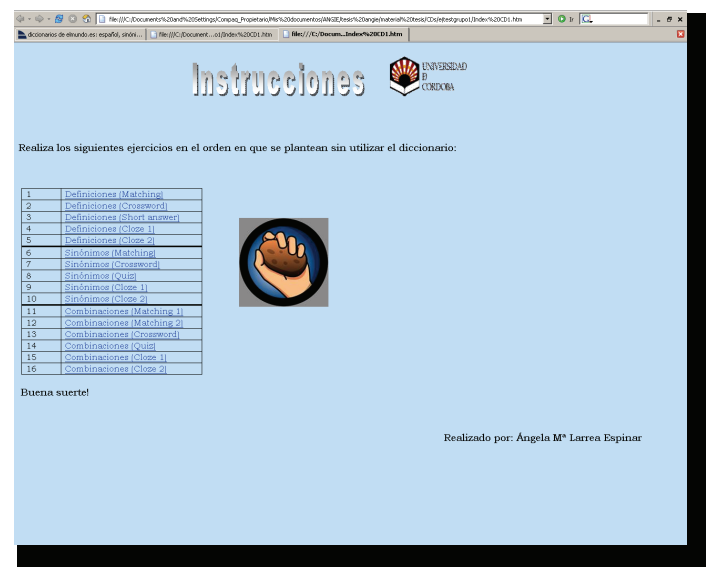

\subsubsection{Evaluación: Cuestionario personal y Autoevaluación}

El cuestionario personal se concibe como un vehículo para que los estudiantes plasmen su opinión personal y reflejen sus impresiones, por lo que es general para todos los grupos experimentales y totalmente anónimo. Está estructurado en dos partes para recoger información cuantitativa y cualitativa, lo que constituye una herramienta muy útil para la investigación educativa (Madrid y Pérez, 2004:130). Para los datos cuantitativos hemos empleado una escala Likert (1932, Likert). En la segunda parte, hemos añadido una serie de preguntas abiertas para que el alumnado se exprese libremente. 
Figura 5: Cuestionario personal cuantitativo y cualitativo.
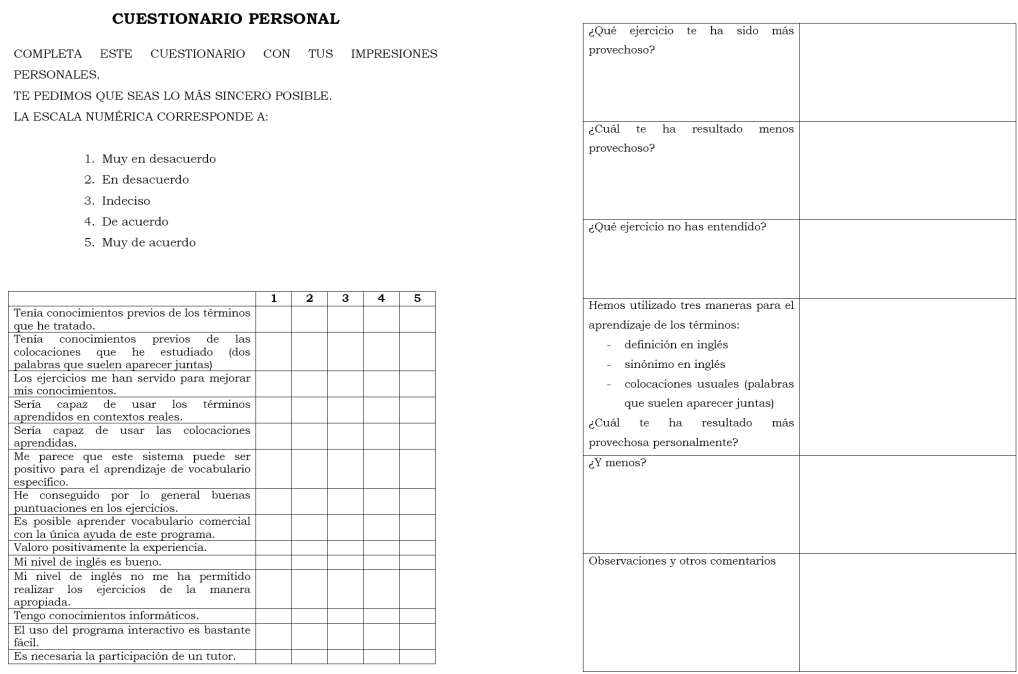

En lo que respecta a la autoevaluación, hemos querido recoger las evaluaciones personales de los alumnos con respecto al nivel de adquisición del vocabulario estudiado. Con este fin, hemos diseñado cuatro tipos de autoevaluación que se corresponden con cada uno de los grupos experimentales. Los estudiantes deberán responder sobre su nivel de conocimiento de los términos tras el experimento así como sobre la retentiva de las colocaciones de los vocablos. 
Figura 6: Autoevaluación del grupo 1.

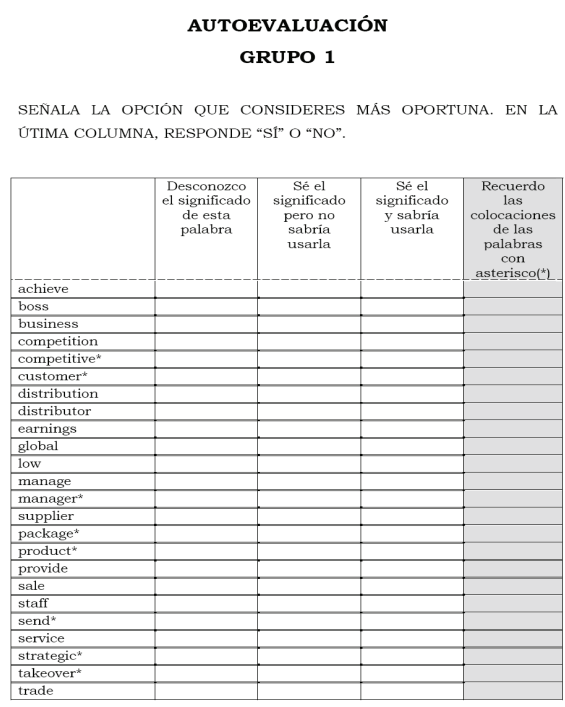

\subsection{Participantes}

Propusimos la participación voluntaria en este experimento a los estudiantes de la asignatura Inglés II, de carácter optativo y perteneciente a la titulación en Administración y Dirección de Empresas de la Facultad de Derecho y Ciencias Económicas y Empresariales de Córdoba ${ }^{15}$.

Contamos, pues, con un grupo de 29 alumnos, a los que se les asignó un grupo de estudio experimental ${ }^{16} \mathrm{y}$, por consiguiente, una secuencia de actividades.

La distribución de los participantes fue la siguiente:

- SECUENCIA 1: 8 personas (Grupo experimental 1)

- SECUENCIA 2: 7 personas (Grupo experimental 2)

- SECUENCIA 3: 7 personas (Grupo experimental 3)

- SECUENCIA 4: 7 personas (Grupo experimental 4)

\subsection{Procedimiento}

Emplazamos a los alumnos por vez primera en una fecha concreta para efectuar el pretest, explicarles el funcionamiento del CD y hacerles entrega del mismo.

${ }^{15} \mathrm{La}$ autora de esta tesis imparte sus clases en esta facultad. Inglés II, que se denomina así genéricamente pero que corresponde a Inglés Comercial II, es la asignatura de la que esta profesora se hace cargo en el segundo cuatrimestre. El experimento fue llevado a cabo en junio del 2007.

${ }^{16}$ Los grupos han sido asignados de manera aleatoria; no obstante, mediante los datos personales requeridos a los estudiantes en el pre-test, nos hemos asegurado de que cada grupo esté integrado por estudiantes de distintos años y de diverso currículo, rendimiento académico y nivel de inglés. 
Acentuamos el hecho de que el CD es una herramienta de estudio personal que ellos debían manejar y que pretendíamos les sirviera para aprender; es decir, en ningún caso se iba a proceder a registrar o comprobar el número de aciertos de los ejercicios virtuales, que sólo tienen valor como instrumento facilitador del aprendizaje.

Se repartió a los alumnos el CD pertinente y se les pidió que hicieran los ejercicios en el orden propuesto y se les dio dos semanas de plazo para que hicieran los ejercicios prácticos.

En el segundo y último emplazamiento, el alumnado completó los post-test, el cuestionario personal y la auto-evaluación (en ese orden).

\section{Resultados}

En lo que respecta a nuestro primer interrogante, una vez analizado el número de aciertos y comparados los resultados del pre-test y del post-test, pudimos verificar que todos los grupos mejoraron considerablemente su conocimiento léxico tras el experimento.

Así, el grupo 1 arrojaba los siguientes datos:

Tabla 3: Resultados Grupo 1

\begin{tabular}{|l||c||c|}
\hline \hline ALUMNOS & $\begin{array}{c}\text { RESULTADO } \\
\text { PRE-TEST }\end{array}$ & $\begin{array}{c}\text { RESULTADO } \\
\text { POST-TEST }\end{array}$ \\
\hline Alumno 1 & $6 / 32$ & $8 / 32$ \\
\hline Alumno 2 & $2 / 32$ & $12 / 32$ \\
\hline Alumno 3 & $21 / 32$ & $22 / 32$ \\
\hline Alumno 4 & $9 / 32$ & $15 / 32$ \\
\hline Alumno 5 & $7 / 32$ & $16 / 32$ \\
\hline Alumno 6 & $6 / 32$ & $14 / 32$ \\
\hline Alumno 7 & $3 / 32$ & $14 / 32$ \\
\hline Alumno 8 & $8 / 32$ & $17 / 32$ \\
\hline
\end{tabular}

Podemos comprobar la comparativa del aprendizaje de este grupo en la siguiente figura:

Figura 7: Comparativa aprendizaje Grupo 1

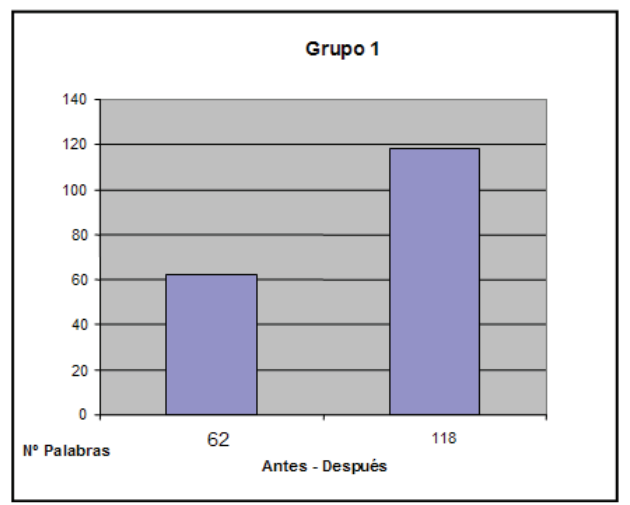


Con relación a los grupos 2 y 3 , el grupo 2 obtuvo 114 palabras correctas en el pos-test (72 antes de realizarlo) y el grupo 3 muestra 120 aciertos, que destacan frente a las 56 palabras correctas del pre-test. La figura correspondiente al grupo 3 incluye la comparativa con respecto al número de palabras total:

Figura 8: Comparativa aprendizaje Grupo 2

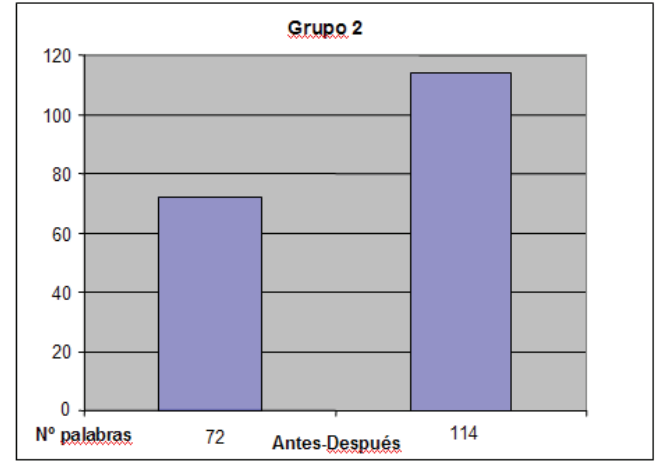

Figura 9: Comparativa aprendizaje Grupo 3

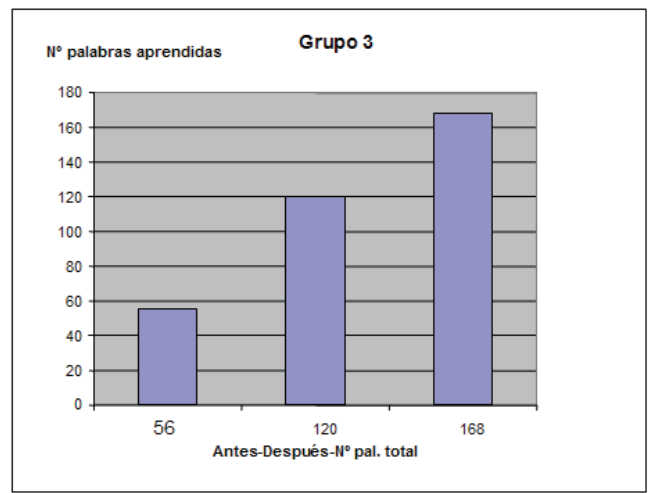


Finalmente, en el siguiente gráfico vemos cómo el grupo 4 también mostró un mayor número de aciertos en el post-test.

Figura 10: Comparativa aprendizaje Grupo 4

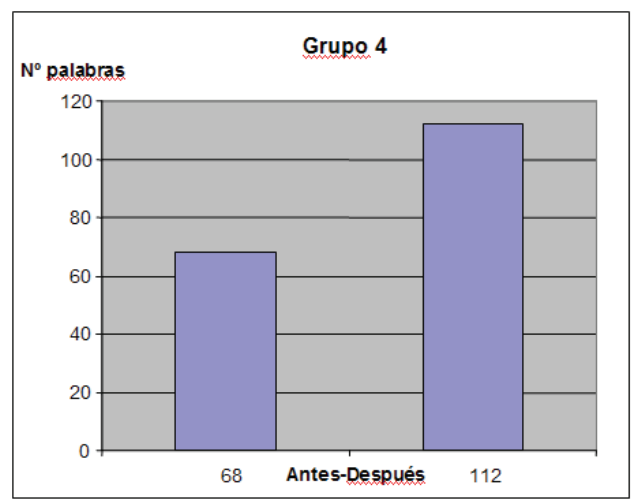

En vista de los resultados obtenidos, corroboramos que se ha llevado a cabo un aprendizaje de vocabulario no sólo a nivel global, sino también a escala personal, ya que las cifras muestran que todos los alumnos aumentaron el número de respuestas correctas en el post-test. Ratificamos, pues, que el aprendizaje de vocabulario ha sido efectivo tanto en grupo como de manera individual. Todo parece indicar que la interactividad y la estructuración metodológica de las actividades han sido acertadas de cara a los resultados obtenidos.

El grupo que más ha destacado en la adquisición de términos ha sido el grupo 3 (64 palabras aprendidas) y el que menos, el grupo 2 (42 palabras). Existe escasa diferencia entre los datos del grupo 2 y del 4 (42 y 44 palabras respectivamente). No obstante, nos han sorprendido las cifras del grupo 1 (56 palabras), ya que contaban con mayor número de términos en su haber (32 frente a las 24 de los restantes grupos $)^{17}$. Pese a la parcialidad del aprendizaje (cada término se ha introducido sólo de una manera) la adquisición léxica del grupo 1 ha sido bastante efectiva, ya que ha superado a los grupos 2 y 4 que contaban con menos palabras en su haber y que habían estudiado los términos desde las tres perspectivas (definición, sinónimo y colocación).

Nuestra segunda cuestión planteaba la idea de que la adquisición léxica a través de conexiones sintagmáticas (colocaciones) fuera más sólida y funcional. En este caso, los estudiantes aprenderían más elementos léxicos mediante colocaciones que términos introducidos de manera aislada (definición o sinónimo). Cotejaremos, por tanto, el porcentaje de adquisición léxica en colocaciones con el de términos presentados de forma aislada.

${ }^{17}$ Debido a que los grupos estaban integrados por alumnos dispares con diferentes aptitudes, objetivos, resultados académicos, intereses, etc. estos resultados no se pueden atribuir a los conocimientos previos o a las aptitudes académicas de este grupo en sí. 
Los resultados de los tests arrojan los siguientes datos para el grupo 1:

Figura 11: Distribución aprendizaje Grupo 1
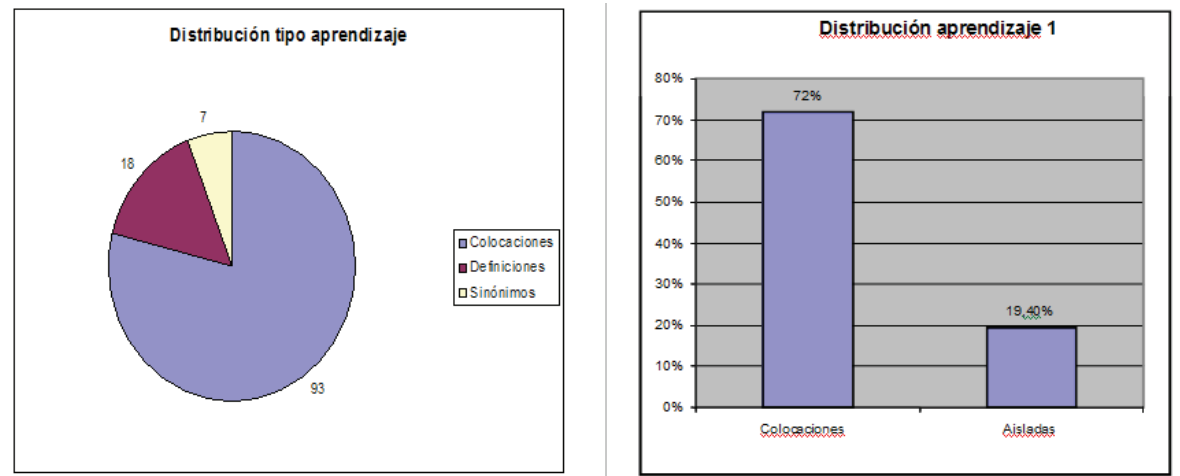

Como muestra el gráfico de la izquierda, estos alumnos aprendieron en total 93 colocaciones, 18 palabras a través de definiciones y sólo 7 de los vocablos expuestos mediante sinonimia. La figura de la derecha recoge esta misma información en porcentajes separando las colocaciones de las palabras aisladas, esto es, que no se introducen a través de conexiones sintagmáticas (lo que engloba las definiciones y los sinónimos).

A continuación, presentamos la distribución del aprendizaje para el resto de los grupos:

Figura 12: Distribución aprendizaje Grupo

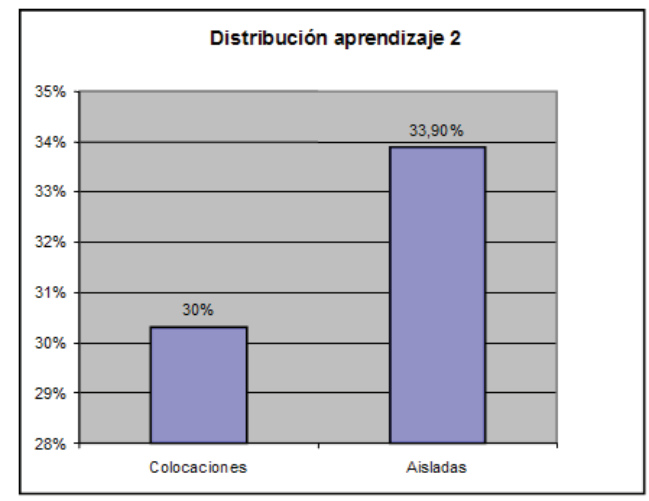


Figura 13: Distribución aprendizaje Grupo 3

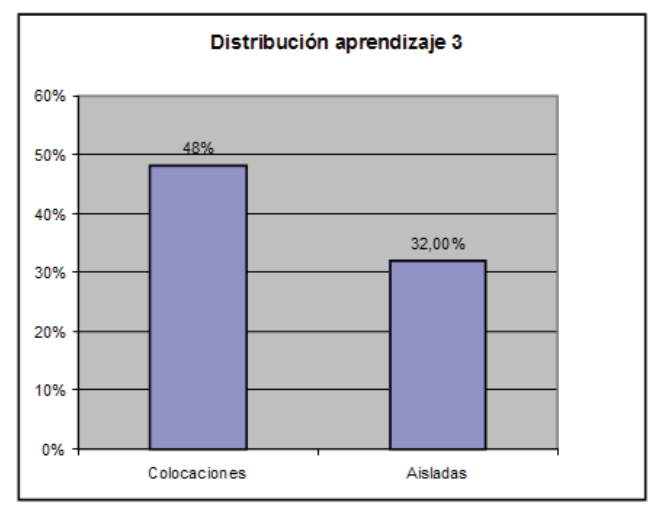

Figura 14: Distribución aprendizaje Grupo 4

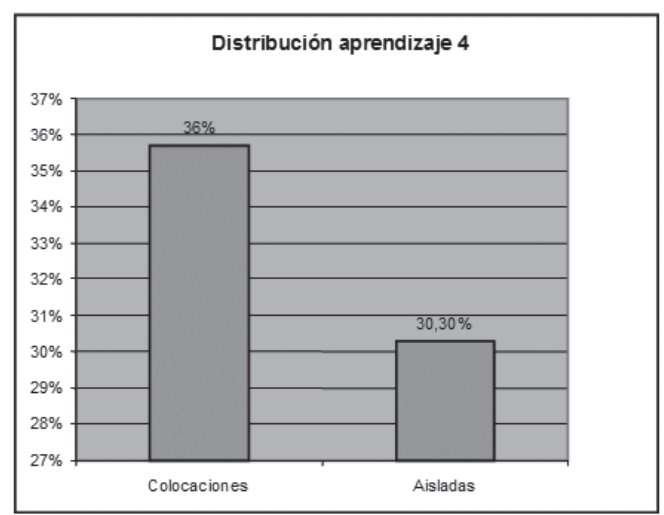

Nuestra segunda hipótesis infería que los grupos participantes aprenderían más palabras presentadas mediante colocaciones como reflejo de la mayor estructuración sintagmática de la L2. Como se aprecia en los gráficos, nuestra segunda hipótesis no se ha demostrado por completo; esto es, no todos los grupos han demostrado un aprendizaje más sólido mediante relaciones sintagmáticas. Así, los datos del grupo 2 indican que estos alumnos han aprendido más palabras de forma aislada que mediante colocaciones (33,9\% palabras aisladas, 30\% colocaciones). Sin embargo, en los casos restantes (grupos 1, 3 y 4) sí que ha habido un aprendizaje más funcional en términos sintagmáticos. A este respecto, el grupo número 1 es el que más ha destacado en la adquisición y además es el que presenta un mayor contraste tanto en el porcentaje de aprendizaje de colocaciones como de palabras aisladas. Esto puede deberse al hecho de que su secuencia de estudio fuera diferente al resto ${ }^{18}$ : mayor número de palabras y cada grupo de términos introducido y estudiado de manera diferente.

${ }^{18}$ Véase el apartado: Secuencia 1 
En lo concerniente al resto de los instrumentos de evaluación, el análisis de los datos cuantitativos del cuestionario personal respalda la primera hipótesis de nuestro experimento, ya que los estudiantes afirman haber mejorado su léxico comercial y manifiestan la utilidad práctica de este programa. Los resultados cualitativos sugieren que el tipo de actividad más útil para el aprendizaje léxico es el de unión de conceptos (Matching), mientras que el menos provechoso es el crucigrama. Las percepciones de los alumnos muestran igualmente que el aprendizaje mediante colocaciones es la manera más provechosa para la adquisición de vocabulario comercial, aunque no existe apenas margen de diferencia con respecto a la definición.

En referencia a la autoevaluación, hemos indagado cuáles son las palabras que los propios estudiantes afirman saber tras el experimento y hemos cotejado los resultados con las que demuestran saber. Así, hemos detectado que las percepciones de los estudiantes, por lo general, son erróneas en cuanto al conocimiento percibido y el real. De esta manera, respecto a los términos aislados, todos los aprendices perciben un conocimiento superior al real. No obstante, en el caso de las colocaciones, el fenómeno se invierte para tres grupos (1, 3 y 4), que tienen un conocimiento mayor del que declaran. La lectura de estos datos merece una consideración extrema: el aprendizaje mediante colocaciones es más perdurable y positivo de lo que perciben los alumnos. Esto reforzaría nuestra conjetura inicial de que el aprendizaje mediante colocaciones sintagmáticas es más sólido.

\section{Conclusiones}

Este estudio experimental ha demostrado que nuestro modelo computacional interactivo mejora la adquisición léxica comercial. En este sentido, vemos cómo el alumnado se puede beneficiar del diseño de material con base cognitiva, esto es, recreando las fases del proceso de adquisición léxica. Así pues, cuando los estudiantes afrontan el aprendizaje de un sublenguaje como el inglés comercial cuya individualidad está más vinculada al componente léxico que al gramatical, este tipo de aplicaciones computacionales resultan extremadamente útiles. Desde una consideración más general, estimamos que este tipo de propuestas didácticas son muy satisfactorias para el alumnado, favoreciendo un aprendizaje significativo y funcional.

Por otra parte, no tenemos certeza de que el aprendizaje mediante conexiones sintagmáticas sea más relevante y efectivo. Esto no invalida la teoría de que el léxico de la L2 se estructure de forma diferente al de la L1 con predominio sintagmático (Wolter, 2001); además, aunque los resultados de la experimentación no son concluyentes, la lectura de los instrumentos evaluativos adicionales así como la información procedente de los datos recogidos de tres de los cuatro grupos experimentales apuntan a la relevancia las conexiones sintagmáticas. Además, el caso extremo del primer grupo ( $72 \%$ colocaciones frente a $19,4 \%$ de términos aislados) nos sugiere que, es posible que, el éxito de la adquisición sintagmática esté relacionado con un gran volumen léxico y la parcialidad del aprendizaje. Así, en situaciones en las que el alumno deba adquirir un número considerable de términos puede que la efectividad del aprendizaje se base en la presentación sintagmática del léxico. A este respecto, interpretamos también que la realidad de la adquisición léxica no está reñida con un aprendizaje parcial, menos integrado y que, presumiblemente, requiere menos tiempo.

La explotación de las incógnitas anteriores así como la imposibilidad de extraer conclusiones generales, debido a la falta de datos concluyentes, son claros indicadores de la necesidad de un mayor número de investigaciones al respecto. 
Como docentes, nuestro estudio pretende sumarse a la exploración de líneas futuras que redunden en la eficiencia y efectividad de la adquisición léxica y, por ende, de la enseñanza de idiomas.

\section{Bibliografía}

Brieger, N. (1997). Teaching business handbook. York: York Associates.

Carter, R. A. (2000). Vocabulary. London: Routledge.

Conklim, K. and Schmitt N. (2008). «Formulaic sequences: Are they processed more quickly than nonformulaic language by native and nonnative speakers? Applied Linguistics, 29.1: 7289

Hutchinson, T. and Waters, A. (1980). «ESP at the crossroads». Swales, J., ed. Episodes in ESP. Oxford: Pergamon, 174-187.

Kitkauskiené, L. (2006). «General English and English for specific purposes» (en línea). Santalka Filologija Edukologija, 14. 2: 88-92. Disponible: http://www.coactivity.vgtu.lt/upload/ filosof_zurn/1-_kitkauskiene_filologija_nr2.pdf (Consulta: julio 2007)

Likert, R. (1932). «A Technique for the Measurement of Attitudes». Archives of Psychology, 140: $1-55$.

Madrid, D. y Pérez, M. L. (2004). «Teacher and student preferences of native and non-native foreign language teachers». Porta Linguarum, 2: 125-138.

Meara, P. (1996). «The dimensions of lexical competence». Brown, G., K. Malmkjær and J. Williams, eds. Competence and performance in language learning. Cambridge: C. U. P., 35-53.

Miller, G. A. (1999). «On knowing a word». Annual Review of Psychology, 50: 1-19.

Nation, I. S .P. (1990). Teaching and learning vocabulary. Massachusetts: Heinle and Heinle.

Nation, I. S .P. (2001). Learning vocabulary in another language. Cambridge: C.U.P.

Nelson, M. (2000). A corpus-based study of business English and business English teaching materials (en línea). Disponible: <http: //users.utu.fi/micnel/business_english_lexis_site.htm> (Consulta: 30 junio 2006).

Pickett, D. (1988). «English in business: knowing and acting». Holden, S., ed. Language and Literature. MEP, 89-95.

Pickett, D. (1989). «The sleeping giant: Investigations in Business English». Language International, 1.1: $5-11$. 